\title{
La orientación vocacional y profesional como estrategia en la comunicación educativa universitaria
}

DOI: $10.46932 / \operatorname{sjdv} 2 \mathrm{n} 1-066$

Received in: November 1st, 2020

Accepted in: December 30th, 2020

\author{
Gustavo Adolfo Santana Sardi \\ Licenciado en Ciencias de la Educación Técnica mención Contabilidad Computarizada \\ Magister en Gerencia Educativa \\ Analista de Promoción y Apoyo al Ingreso \\ Universidad Técnica de Manabí \\ E-mail: gusansar@hotmail.com \\ Jhimmy Andrés Gutiérrez Santana \\ Licenciado en Ciencias de la Educación especialidad Psicología y Orientación Vocacional \\ Magister en Docencia e Investigación Educativa \\ Docente Agregado Tiempo Completo (Facultad de Ciencias Sociales y Humanísticas) \\ Universidad Técnica de Manabí \\ E-mail: jhimmyg@yahoo.com \\ Francisco Antonio Mawyin Cevallos \\ Licenciado en Ciencias de la Educación especialidad Psicología y Orientación Vocacional \\ Magister en Orientación Educativa Vocacional y Profesional \\ Doctor dentro del programa de Doctorado en Educación \\ Docente Auxiliar Tiempo Completo \\ Universidad Técnica de Manabí \\ E-mail: franciscomawyin2016@gmail.com

\section{Pedro Alejandro Escobar Palma} \\ Licenciado en Ciencias de la Comunicación mención Periodismo \\ Magister en Periodismo mención en Periodismo Digital y Gestión de Proyectos Multimedia \\ Periodista del departamento de redacción \\ Medios Ediasa \\ E-mail: pedroescohbk@gmail.com
}

\section{RESUMEN}

Este documento tiene como propósito influenciar las intenciones vocacionales que la persona requiere, el conocimiento de sí mismo, para poder implementarlos en sus planes de estudio, de las propuestas de trabajo, de las competencias que debe desarrollar para alcanzar un buen desempeño lo cual le permitirá tomar las decisiones que considere de acuerdo a sus capacidades y aptitudes para ubicarse en el contexto sociallaboral. La orientación vocacional y profesional adquieren un significado muy alto en las actividades académicas dando paso a adquirir estrategias en una comunicación educativa adecuada en estudiantes, siendo este el dinamismo que estas presentan para ir respondiendo a los cambios sociales, y que se reflejan en el ambito profesional, además porque se debe considerar el carácter solidario y humanista que encierra cualquiera de sus perfiles profesionales. Para esto se realiza un análisis teórico acerca de la orientación vocacional y profesional como estrategia en la comunicación educativa en el contexto de la formación profesional, de tal forma que hace énfasis de que dicho enfoque se ubica en la formación integral del individuo, expresado en las capacidades conceptuales, procedimentales y actitudinales que han de construirse en el estudiante para ser considerado una persona competitiva. 
Palabras claves: orientación vocacional y profesional, estrategia, comunicación educativa universitaria.

\section{INTRODUCCION}

La sociedad actual demanda un sujeto capaz de conocerse a sí mismo y de conocer qué oportunidades laborales y académicas le son más dadas de acuerdo a sus aptitudes y actitudes personales, si tenemos en cuenta que las vocaciones y los valores se orientan, forman y educan, entonces, la orientación vocacional no es solo una intervención puntual en algún momento de la vida de un individuo, es más bien un proceso continuo en el tiempo, que acompaña al individuo en su formación durante toda la vida, de manera que conozca y tome decisiones para construir su propio conocimiento de acuerdo a su vocación.

Desde la orientación, la transición se concibe como una oportunidad de desarrollo personal, a través de la cual, la persona debe aprender a gestionar e integrar los cambios que se producen en él mismo y en su entorno con la pretensión de construir, como agente activo, su propio proyecto vital y así, planificar y afianzar su desarrollo personal y profesional, además de ajustarse a la rapidez y vertiginosidad de los cambios que acontecen en la sociedad y que operan en el ámbito social, formativo y laboral. (González y González, 2015, p. 30)

De igual forma, para realizar una elección vocacional acertada, se requiere de un determinado nivel de desarrollo de la madurez vocacional que permita establecer objetivos profesionales que sean plenos para la persona y para la demanda del mercado laboral (López Fernández \& Sánchez Herrera, 2018). Por lo tanto, teniendo en cuenta que los adolescentes o adultos aún están en un proceso madurativo en desarrollo, necesitan de otros individuos con mayor experiencia que orienten y guíen la elaboración de su proyecto de vida, lo que conlleva la implicación de padres o representantes de familia, docentes, orientadores, entre otros.

Educación y comunicación son dos términos que pueden ser entendidos de muy diversa forma; y, según se usó de los medios en la enseñanza. Con todo el riesgo de una simplificación esquemática, se puede distinguir entre dos modos de entenderlos; y la opción por uno de ellos atravesará y permeará toda la práctica educativa.

En los sistemas de actividad y comunicación en que se desarrolla el sujeto se crean las necesidades para estimular o no las particularidades subjetivas de la personalidad. La persona va configurando su subjetividad sobre la base de aquello que necesita para individualizar su expresión y alcanzar sus objetivos. La importancia que la universidad tiene en la formación de los alumnos en los medios de comunicación, viene no sólo porque generen actividades específicas formativas, sino también por el modelo social, cultural y curricular en el que se desarrolle la universidad. Así, la forma en que la universidad socializa a los estudiantes, los métodos y estrategias docentes que utiliza en su formación, parecen también determinar el tipo de interacción que luego de eso establecerán con los medios. Una universidad donde el método de 
enseñanza es autoritario y se socializa al alumno para obedecer las normas, tiende a formar alumnos que consumen de manera no crítica la información que les llega a través de los medios de comunicación.

\section{DESARROLLO}

Según menciona Santana Sardi, G. A y Vigueras Moreno (2019) la creación de las bases económicosociales y las exigencias actuales de su desarrollo, requieren continuar elevando la calidad del trabajo educacional, en el cual constituye un punto importante el desarrollo de intereses en los estudiantes.

Muchas veces uno de los problemas de la educación no radica en lo que se le comunica y transfiere al alumno, sino precisamente en lo que deja de comunicársele, y que puede impedir el desarrollo de futuras habilidades y estrategias donde la interacción con determinados medios tendrán una elevada importancia en nuestra sociedad futura. Como se pudo investigar de diversos autores, las actitudes que tenemos hacia los medios (Castaño, 1992; Cabero y otros 1993), condiciona la interacción que se establezca con los mismos, y viene claramente estructurada por las experiencias en la vida universitaria y familiares que el alumno tenga con los mismos.

Ahora bien, no podemos olvidar que el papel fundamental en esta formación con los medios de comunicación, lo tiene el docente. Éste cabe recalcar que influye tanto con las actitudes que tenga hacia los medios, como con los usos y propuestas que haga con ellos en el aula. No podemos tampoco olvidar, que la educación en cualquier medio de comunicación e información, pasa necesariamente por la posibilidad de que la persona se convierta en emisor de mensajes. Al respecto, ya en otro trabajo (Cabero, 1991) señala que en el terreno educativo pueden realizarse tres tipos de producciones: profesor y/o los alumnos, profesionales de los medios, e instituciones educativas. Las primeras, que son las que aquí nos interesan tienen una serie de ventajas de la que podemos resaltar la posibilidad que el alumno tiene de introducirse en el conocimiento técnico y sémico del medio que se utilice para la elaboración de los mensajes.

La preparación del profesor es decisiva para enfrentar los desafíos que entrañan orientar. Se impone la necesidad de dominar variados métodos y procedimientos para realizar esta actividad como lo demandan los estudiantes, al atender el grado de motivación, interés, éxito, progreso, dificultades, para propiciar, de manera sistemática, una OP más eficaz, de mayor calidad en función de la individualidad. (Brito, 1990).

Para emprender el estudio de la comunicación como problema y de la teoría de la comunicación como ciencia, es necesario establecer previamente con claridad, una serie de aspectos de carácter conceptual en los cuales se sustenta todo el sistema de conocimientos de esta rama. Diferentes son los trabajos relacionados con la comunicación de manera general que aparecen desde Platón y Aristóteles sobre el lenguaje, continuados en Cuba por diferentes pedagogos tales como Luz y Caballero (1825), Varela (1830) y Martí (1880), entre otros estudios que constituyen referentes para la presente investigación. Los anteriores antes mencionados han sistematizado y profundizado en las diferentes posiciones teóricas. Hall Walfriman 
y Sheflon (1980) expresan que la comunicación concebida como la matriz de todas las actividades humanas, como proceso permanente y circular de interacciones en los que cada hombre participa.

La educación, inmersa en un continuo perfeccionamiento, exige respuestas concretas de los docentes, a partir de su práctica profesional en los diferentes niveles de educación, con el objetivo de lograr el mejoramiento del proceso de formación integral de las nuevas generaciones. (Martín, Juárez, Lorente, Cusó, Morga, \& Clares, 2017).

En cierto momento González Rey F.(1985) dice que la comunicación es un proceso esencial de toda la actividad humana, ya que se basa en la calidad de los sistemas interactivos en que el sujeto se desempeña, y además tiene un papel fundamental en la atmósfera psicológica de todo grupo humano.

Para B. F Lomov (1989: 293) la comunicación es la interacción de las personas que entran en ella como sujetos, no sólo se trata del influjo de un sujeto con otro, aunque esto no se excluye, sino de la interrelación entre ambos. En ella se lleva a cabo un intercambio de actividades, representaciones, ideas, orientaciones, intereses, etcétera, se desarrolla y manifiesta el sistema de relaciones sujeto-objeto, necesitándose como mínimo para su realización, dos personas, cada una de las cuales actúa como sujeto.

El autor Lomov (1986), asume la comunicación educativa como un proceso en que se crece dentro de ella, por lo tanto para crecer es necesario establecerlo. La comunicación como un proceso complejo, por lo que ser un buen comunicador no resulta nada fácil, aunque es posible desarrollarla a partir de un entrenamiento y de la actividad que asuma el docente. La comunicación es un proceso en extremo activo en el cual los elementos que participan en ella siempre lo hacen en condición de sujetos de ese proceso. En el proceso de la comunicación las personas se relacionan tanto por la vía verbal como la no verbal. Este autor además, asume que la comunicación educativa se define como la interacción entre los docentes y los estudiantes.

De acuerdo a lo investigado aparece como un aspecto de mucha importancia para el trabajo de la comunicación del docente con los estudiantes los criterios abordados por González Rey, Fernando (1995):

1. El aprendizaje como proceso de la comunicación.

2. La construcción del conocimiento a través del diálogo.

3. La evaluación como proceso de la comunicación.

4. La comunicación con los padres como aspecto esencial de la comunicación educativa.

5. Significación de la comunicación individual con el escolar.

Según ( MINEDUC, 2015) para poder trabajar el tema de la OVP, es importante que partamos de una definición general sobre este concepto:

La orientación vocacional y profesional (OVP) comprende un conjunto de acciones de acompañamiento (educativo-psicológico-social) y asesoramiento (individual y grupal) dirigido a las y los 
estudiantes de una institución educativa para que, de manera individual y con base en el autoconocimiento y la información disponible, tomen decisiones vocacionales y profesionales adecuadas como parte de la construcción de su proyecto de vida.

Esta ha sido priorizada de diversas maneras en la actualidad en varios sistemas educacionales de Latinoamérica y a nivel global, como uno de los objetivos más importantes del trabajo educacional y se encuentra dirigida a la preparación estudiantil relacionada con su incorporación al complejo mundo de la producción y de los servicios, de modo tal que sean capaces de realizar una selección profesional consciente, fundamentada en sus intereses y posibilidades reales en concordancia con las necesidades del país. (Pérez, Filella, \& Bisquerra, 2009). La Educacion es uno de los espacios más importantes y decisivos de formación vocacional y profesional en donde las y los estudiantes contarán con un sinnúmero de estímulos y experiencias que les permitirán explorar sus gustos, habilidades, intereses, capacidades y deseos en relación con lo que quieran hacer en la vida.

Al hablar de Orientación Vocacional, se refiere también a la educación de una profesión y dentro de esto estamos hablando de Pedagogía, puesto que la Pedagogía es una disciplina que tiene por objeto de estudio la educación, la cual no sólo se enfoca de la enseñanza aprendizaje o de la transmisión de conocimientos que se le proporciona a los estudiantes por parte del docente dentro de un aula de clase, sino que también abarca muchos aspectos donde se desenvuelve la persona, como escolar, social, laboral, familiar, entre otras; por tanto la pedagogía influye en la formación del hombre como persona y profesional a través de la educación, reforzada en casa y después en la escuela, teniendo como base el vínculo de enseñanza-aprendizaje, docente-estudiante pero siempre de manera constructiva.

Cuando pensamos en la OVP, debemos tomar en cuenta que está conformada por dos aspectos que se conectan y son igualmente importantes; El componente vocacional de la OVP tiene que ver con el conjunto de gustos, intereses, conocimientos y habilidades que determinan una tendencia en la persona hacia el desarrollo de cierta/s actividad/es a lo largo de la vida y con proyección hacia el futuro, en el contexto de la realidad en que se desarrolla y el componente profesional de la OVP tiene que ver con las decisiones que adoptará la o el estudiante en el ejercicio de una actividad ocupacional o laboral puntual.

A pesar de surgir diversos criterios, nuevas formas organizativas y estrategias para elevar la calidad de la formación, desarrollo y consolidación de intereses, conocimientos y habilidades profesionales, basadas fundamentalmente en enfoques psicológicos y pedagógicos; en la práctica educativa se han detectado insuficiencias que parten de los resultados obtenidos en la aplicación de instrumentos y métodos de investigación, es la expresión resultante de la actuación de docentes y educandos del nivel preuniversitario en las diferentes situaciones en las que se ven involucrados como actores en el proceso de orientación profesional. (Valdés, Valdés, \& Fernández, 2016). 
La OVP es un proceso que puede ser comprendido simultáneamente desde diversas perspectivas. Es decir, cada vez que llevamos a cabo una iniciativa de OVP, esta puede ser mirada o analizada desde diferentes enfoques:

a. Enfoque de derechos: Aplicar un enfoque de derechos significa, antes que nada, reconocer que todo niño, niña y adolescente cuenta con derechos humanos, independientemente de su sexo (género), orientación sexual, edad, etnia, cultura o condición social. Es deber del Estado, así como del sistema de educación ecuatoriano, garantizar su aplicación y cumplimiento.

b. Enfoque de género: El género es una categoría de análisis que impregna todas las relaciones humanas y que permite distinguir cualidades, capacidades, mandatos, roles y estereotipos que se asignan a hombres y mujeres desde una perspectiva social y cultural, que se van gestando desde etapas tempranas del desarrollo humano y que también permiten reconocer y analizar preponderantemente, las causas vinculadas a un trato discriminante, desvalorizante y peyorativo contra lo que representa lo femenino o lo masculino

c. Enfoque intercultural: Es un enfoque que no sólo se reduce al contacto cultural o al reconocimiento de culturas diferentes, sino que acapara también lo diverso de las cosmovisiones, formas, opciones, concepciones y prácticas de vida implícitas en el acto de comprender y respetar al otro. El reconocimiento de la coexistencia de diversidades culturales en las sociedades actuales debe ser llevado con base en el respeto de sus derechos humanos y derechos como pueblos, según lo manifiestan (Faúndez, Alejandra y Weinstein, Marisa, 2012)

d. Enfoque intergeneracional: Así también, el enfoque intergeneracional se orienta a reducir las desigualdades existentes en las relaciones que mantienen las personas adultas y las y los jóvenes para que estos puedan determinar con autonomía qué quieren, para qué, cómo, dónde y con quién lo quieren hacer.

e. Enfoque de bienestar: El incluir este enfoque permite que en la configuración de las directrices para la OVP con énfasis en proyectos de vida se establezcan estrategias enmarcadas en la comprensión de los diferentes procesos psicológicos (afectivos, emocionales y subjetivos) que acompañan el desarrollo de niños, niñas y adolescentes, así como la inclinación y escucha activa de la situación personal de la o el estudiante, su contención psicológica-emocional y el mejoramiento de la calidad de la relación entre la persona adulta a cargo y la o el estudiante.

f. Enfoque inclusivo: La inclusión educativa es inherente al principio de Educación para todos, y demanda un cambio importante tanto en la forma de concebir y desarrollar la educación en sus aspectos curriculares, como en la actitud de los maestros, maestras y autoridades educativas frente a los estudiantes. Y; 
g. Enfoque pedagógico: se fundamenta en el concepto de Educación para la vida, que posibilita la formación y el desarrollo humano desde una visión integral, en donde: los estudiantes vivan experiencias esenciales y afines a los intereses, aptitudes y actitudes que fortalezcan la toma de decisiones personales; y los educadores debatan las experiencias observadas, vividas y sistematizadas a la luz de teorías e investigaciones científicas, culturales, socio-políticas y tecnológicas contemporáneas, para desarrollar la capacidad de plantearse interrogantes alrededor de problemas prácticos y teóricos para que se conviertan en desafíos que provoquen en ellos la necesidad de investigar y diseñar soluciones creativas a las necesidades educativas prioritarias.

Las concepciones de L. S. Vigotsky rompieron con las conceptualizaciones tradicionales sobre el diagnóstico, con la aparición ya de su denominada ZONA DE DESARROLLO PRÓXIMO, es decir, la capacidad del estudiante universitario para asimilar los niveles de ayuda y su posibilidad real de independizarse, realizando acciones por sí solo a partir de la experiencia anterior y el desarrollo de sus conocimientos, hábitos y habilidades que adquieren también a partir de la interrelación colaborativa con sus coetáneos, facilitando la labor de motivación y Orientación Profesional.

La estrategia es diseñada, orientada y evaluada con objetivos, acciones y tareas que son basadas en la flexibilidad y por la factible evaluación de la misma. Para esto, se debe cumplir ciertas metas que corresponden con el estudio de distintas definiciones, así como se tienen presentes elementos vitales para su desarrollo.

Es necesario destacar que la estrategia de Orientación vocacional y profesional, cumple la función pedagógica, ya que va a contribuir al perfeccionamiento de este proceso y al proceso educativo-formativo de los estudiantes.

Para fundamentar de forma general la estrategia en la comunicación educativa, la cual declara las estructuras y partes que posee. En el desarrollo de la actividad estratégica que se asume, se tienen en cuenta las potencialidades afectivas entre los docentes y estudiantes. Por esta causa, se declaran en el proceso de desarrollo estratégico los siguientes valores compartidos:

- Motivación y estimulación para el desarrollo de las acciones correspondientes para todos los implicados.

- Capacidad crítica y autocrítica en el desempeño de cada actividad.

- Elevación en el nivel de conciencia respecto a la necesidad de transformar el proceso de orientación profesional y formación vocacional en la educación preuniversitaria.

Respecto también con la educación, se involucra en un continuo proceso para su mayor efectividad, exigiendo respuestas concretas de los docentes, a partir de su práctica profesional en los diferentes niveles 
de educación, con el objetivo de lograr el mejoramiento del proceso de formación integral de las nuevas generaciones. (Martín, Juárez, Lorente, Cusó, Morga, \& Clares, 2017).

Los estudiantes por alta de conocimientos que le ayuden a la orientación sobre las diferentes profesiones y por este motivo, no están motivados para el estudio de las mismas, así como la falta de preparación de los profesores y la familia que no contribuye al logro de los objetivos deseados, por lo que se plantean como limitaciones:

- Insuficientes acciones estratégicas en la orientación vocacional que permitan cumplir con el ingreso y calidad de los bachilleres.

- Falta preparación en los elementos teóricos y metodológicos que sustentan la orientación vocacional en directivos y docentes.

- Falta sistematicidad y carácter de sistema de las acciones estratégicas de la orientación vocacional.

- Falta de integración y compromiso de los agentes educativos que intervienen en los procesos de orientación vocacional. (Riesgo, Jayme, \& Ordaz, 2018).

Como objetivo general de la estrategia consiste en la implementación de un conjunto de acciones estratégicas, estructuradas en una lógica de trabajo y encaminadas a favorecer el proceso de orientación vocacional y profesional a los estudiantes que ingresan a la enseñanza media superior.

Es así que la orientación vocacional debe ser para los estudiantes de gran prioridad en la decisión de su futuro profesional, puesto que la importancia de la orientación vocacional en la educación media y universitaria se basa en los aspectos pasados que puede inmiscuir la carencia y la necesidad económica, política y cultural de nuestro país donde se generaría un proceso de orientación vocacional y profesional pertinente; dado que el resultado de planificar y construir esta actividad puede reducir de manera sustancial el tiempo que a un estudiante le costaría reconocer en qué área, carrera o profesión tiene ventaja comparativa en relación con su grupo.

De esta manera, (Blanco, 2012) afirma que una de las principales características en la Orientación es que su desarrollo se hace en forma sistemática de acuerdo a los factores internos que demuestra cada estudiante. En forma de estrategia mediante un proceso en que se lo involucra en el crecimiento de habilidades y potencialidades desarrollando el perfeccionamiento que ha de verse en una doble intención Una de ellas es su perfeccionamiento como ser humano, como persona adulta susceptible de desarrollo, tanto en sus conocimientos, habilidades y capacidades, como en sus comportamientos, motivaciones, intereses, autovaloración y autodeterminación. La otra intención se enfoca al perfeccionamiento de su capacidad profesional.

Tal como lo indica estos autores, se orienta y expresa que las instituciones universitarias pueden y deben acompañar el procesos de orientación profesional que se gesta en sus inicios desde los colegios, en la 
enseñanza básica superior, cuando el estudiante se encuentra a punto de solicitar sus ingreso al régimen universitario. (Santana, Vélez, Sampedro, \& Delgado, 2020)

Como lo menciona en su artículo (Alcívar, Santana, Zambrano, Sardi, \& Riverón, 2020; p. 911), Para la orientación profesional se conocen diferentes métodos que concretan su desarrollo en el proceso formativo, producto a que estos "(...) son las vías que se siguen para lograr el objetivo educativo planteado por la sociedad y las tareas específicas a desarrollar en condiciones determinadas por el proceso educativo." (Álvarez, et. al. 1990 p. 38).

Esto supone que para conocer mejor a la orientación profesional en estudiantes universitarios se necesitan de distintos métodos que le facilitaran en su formación profesional donde habrá un resultado favorable para la toma de su decisiones beneficiando así a la comunidad y tendrá la suficiente capacidad para resolver sus tareas diarias y a lo largo de su vida estudiantil y profesional.

A través de la revisión de diversos autores que han trabajado en los últimos años sobre la comunicación educativa como son: (Falconí, 2017); (González, 2017); (Hernández, Orrego, \& Quiñones, 2018); (Mañas, \& Roig, 2019); (Narváez, 2019); (Moscoso, \& Iglesias, 2019); entre otros, es posible identificar distintas perspectivas alrededor de este campo, enfoque o espacio interdisciplinario en el que convergen principalmente fundamentos teóricos de la comunicación y de la educación. La comunicación educativa, como concepto, radica en el vínculo indiscutible entre comunicación y educación, la utilización de medios de comunicación y tecnologías digitales en procesos formativos, la preparación de educadores en la incorporación de recursos mediáticos para la enseñanza y el aprendizaje, incluso la pedagogía de la comunicación y, más recientemente, paradigmas de comunicación para el desarrollo y la transformación de realidades sociales.

En la educación tanto en conceptos generales como mayor puntualidad, se requiere de hacer el uso de la comunicación y, es conocido que no siempre la comunicación educa o busca educar. Por lo tanto, en diversos espacios de enseñanza y aprendizaje, en la cual el espacio virtual está presente como vía de desarrollo de la formación, donde media la distancia física entre profesor y estudiante, el papel de la comunicación educativa es aún más importante.

Mario Kaplun (1983) sintetizó tres modelos básicos de comunicación educativa, que si bien hoy podemos tener una perspectiva distinta para analizarlos han sido instrumentos útiles para caracterizar la relación profesor- alumno. La realidad social que se vive en la universidad, las interacciones entre los actores del proceso educativo, las cosmovisiones por parte de estos actores, los conflictos, las influencias de la clase social, el lugar en que se vive, las expectativas de vida, la subcultura a la que se pertenece, los conflictos que se desarrollan, las normas que se practican, las creencias, los hábitos, las valoraciones de la cultura ideal y material, las pautas de crianza, las pautas de socialización, en fin, todos los rasgos culturales que se dan en la práctica pedagógica constituyen la unidad principal que debe ser extraída 
con el fin de comprenderla y transformarla en acción deliberada de los diversos actores implicados en el proceso (Parra, 1998).

Entre otros aspectos durante la puesta en práctica de la comunicación educativa, se manifiestan el uso adecuado del lenguaje como oportunidad y adecuación, ritmo y cadencia que lo haga asequible, saber hablar y saber escuchar, potenciar el diálogo constante en cada clase, orientarse al interlocutor, ponerse en el lugar de los demás, estimular la participación, respetar y aceptar a los otros tal y como son, buscar soluciones educativas a los conflictos, establecer un clima psicológico favorable; además de favorecer y organizar el trabajo conjunto.

Continuando, Ojalvo, V. (1999) que presupone que el educador debe partir de un autoanálisis de sus propias dificultades y limitaciones para la comunicación, así desarrollar habilidades para mejorar cada día la sociabilidad y su empatía con sus alumnos, la confianza de estos, la creatividad para propiciar el intercambio de conocimientos entre los estudiantes y sobre todo aumentar la percepción rápida de lo que pueden manifestar sus educandos.

En un sentido amplio y general, y siguiendo lo expresado por Trilla (1993:22), se considera comunicación educativa a "cualquier proceso de comunicación que genere algún efecto educativo". Si esta se produce intencionalmente, de acuerdo a algún propósito pedagógico, y recurriendo a formas y códigos propios, se estará hablando de procesos de comunicación educativa en lo formal y no formal. En cambio, si el proceso comunicativo se establece y desarrolla sin haberse estructurado en base a una intencionalidad educativa, pero, no obstante, se producen efectos educativos, entonces se hablará de comunicación educativa informal.

(De Monte, 2013), en este sentido, el diseño de estrategias para la enseñanza debe ir orientadas a un propósito específico tomando en cuenta las necesidades y características del grupo. En el ámbito de las teorías pedagógicas, diversidad de autores han desarrollados sus visiones sobre las estrategias que debe desarrollar el docente en su práctica educativa, concretamente, orientadas hacia el proceso de enseñanza y aprendizaje.

\section{CONCLUSIONES}

El objetivo fundamental de este artículo fue comprender y analizar los factores en la orientación vocacional y profesional como estrategia en la comunicación educativa en estudiantes universitarios, desde diferentes enfoques. De los temas adjuntos se concluye que la visión personal y profesional de los estudiantes universitarios está determinado por un contexto en el que abarca la participación de los docentes como orientadores y los estudiantes como receptores del aprendizaje; además, siendo de estos determinantes sobre las estrategias comunicativas que se dan cotidianamente en el interior del núcleo de formación profesional con antecedentes familiares. 
Las aproximaciones interpretativas contribuyen significativamente a la investigación en el aula, y esto se da por varias razones, entre las cuales destacan la consideración de las aulas como espacio social y cultural organizado intencionalmente para propiciar el proceso de enseñanza-aprendizaje; su interés en la búsqueda de lo menos obvio, de las relaciones invisibles en las interacciones entre profesores y estudiantes; y, por último, el énfasis dado a la interpretación, significación y construcción de saberes desde el punto de vista de los participantes.

La comunicación educativa entendida como modalidad de la comunicación, ocurre precisamente durante el proceso de enseñanza-aprendizaje y se encamina a la creación de un clima favorable entre el profesor y los estudiantes, que propulse la optimización de la actividad de estudio. A través de ella se logra incentivar en los jóvenes, actitudes e intereses positivos hacia los contenidos, el aumento de la motivación cognitiva y la transformación de los motivos externos en internos.

Este tema de la comunicación educativa, a diferencia de otras, no es aleatoria ni casual, al menos en lo concerniente a lo formal y no formal, ya que obedece a una intencionalidad. Por lo tanto, responde a los intereses y necesidades que poseen sobre un tema, tanto el emisor, en este caso el docente, como el receptor, es decir, el estudiante. Se deben precisar los contenidos y objetivos de los mismos, ya sean de tipo formativo o informativo.

La estrategia planteada permite organizar rigurosamente el trabajo de orientación vocacional y profesional de manera secuencial. Cuando se conocen diversas conceptualizaciones permitieron analizar la calidad en forma comparativa, se llega a la conclusión que con su implementación se alcanza un significativo muy favorable, porque los estudiantes demuestran muy buena preparación y motivación para su vida personal y profesional, así como, correspondencia entre el grupo de carreras donde se encuentran y sus potencialidades para optar por estas en función de aquellas profesiones necesarias para su desenvolvimiento. 


\section{BIBLIOGRAFIA}

Alcívar, W. R. M., Santana, J. A. G., Zambrano, N. L. Z., Sardi, G. S., \& Riverón, J. L. N. (2020). Propuesta de guía para favorecer la orientación profesional para su ingreso a la educación superior ecuatoriana. Polo del Conocimiento: Revista científico-profesional, 5(10), 903-918.

Álvarez Aguilar, N. et. al. (1990). La orientación profesional de los escolares a través del proceso docente. Ponencia. Pedagogía 90'. La Habana, 1990. (Manuscrito en el CDIP Nacional)

Blanco, M. C. (2012). Reflexiones y experiencias a propósito de la orientación vocacional en Bogotá. Bogotá: Universidad de los Andes.

Brito Hernández, H (1990). La efectividad de la motivación. En Ciencias Pedagógicas. no. 20. La Habana, 1990, pág 55-56.

CABERO, J. (1991): «Producción o producciones audiovisuales en el terreno educativo?», en El siglo que viene, 11, 19-22.

CABERO y OTROS (1993): «Esfuerzo mental y percepciones sobre la televisión/vídeo y el libro», en Bordón, 45, 2, 143-153.

DeMonte, J., High-Quality Professional Development for Teachers: Supporting Teacher Training to Improve Student Learning, Center for American Progress, 28 (2013)

Falconí Asanza, A. V. (2017). Inclusión de la tecnología móvil de información y comunicación educativa como estrategia pedagógica. Revista Universidad y Sociedad, 9(4), 82-89.

Faúndez, Alejandra y Weinstein, Marisa (UNFPA, PNUD, ONU MUJERES). Ampliando la mirada: la integración de los enfoques de género, interculturalidad y derechos humanos. Oficina y Centro Regional para América Latina y El Caribe, LACRO, TACRO, ACRO. Santiago de Chile, 2012, pág. 23

González, C. \& González, N. (2015). Enseñar a transitar desde la Educación Primaria: el proyecto profesional y vital. Revista Electrónica Interuniversitaria de Formación del Profesorado, 18(2), 29-41.

González-Morales, L. (2017). Metodología para el diseño instruccional en la modalidad b-learning desde la Comunicación Educativa. Razón y Palabra, 21(3_98), 32-50.

GONZÁLEZ REY, FERNANDO. (1985). Psicología de la personalidad. Editorial: Pueblo y Educación, La Habana.

GONZÁLEZ REY, FERNANDO (1990). Psicoterapia y personalidad; algunas reflexiones. En: Revista Cubana de Psicología, Vol. VII, No. 2, p. 117-127, La Habana.

Hernández, R. M., Orrego Cumpa, R., \& Quiñones Rodríguez, S. (2018). Nuevas formas de aprender: La formación docente frente al uso de las TIC. Propósitos y Representaciones, 6(2), 671-685.

LOMOV, B. (1989). El problema de la comunicación en psicología. Editorial de Ciencias Sociales, La Habana.

LOMOV, B. (1983). Comunicación y regulación social de la conducta del individuo. En: Aspectos socio psicológicos de la comunicación, p. 3-32. Facultad de Psicología. Universidad de La Habana, La Habana 
López Fernández, M., \& Sánchez Herrera, S. (2018). Relación entre la madurez vocacional y la motivación hacia el aprendizaje académico. International Journal of Developmental and Educational Psychology, 1(1), 21-30.

Mañas Pérez, A., \& Roig-Vila, R. (2019). Las Tecnologías de la Información y la Comunicación en el ámbito educativo. Un tándem necesario en el contexto de la sociedad actual.

Martín, M. S., Juárez, M. M., Lorente, C. G., Cusó, J. P., Morga, N. G., \& Clares, P. M. (2017). Satisfacción vocacional con la elección del ciclo de Formación Profesional. Revista de estudios e investigación en psicología y educación, (7), 36-40.

Ministerio de Educación de Ecuador. Acuerdo Ministerial 332-13. Guía metodológica para la construcción participativa del Código de Convivencia Institucional. Quito, Septiembre 2013.

Kaplun, Mario (1983) El comunicador popular, Buenos Aires: Humanitas

Martín, M. S., Juárez, M. M., Lorente, C. G., Cusó, J. P., Morga, N. G., \& Clares, P. M.

(2017). Satisfacción vocacional con la elección del ciclo de Formación Profesional. Revista de estudios e investigación en psicología y educación, (7), 36-40.

MARTÍ PÉREZ, JOSÉ. (1990). Ideario pedagógico. Editorial: Pueblo y Educación, La Habana.

Ojalvo, V. Aspectos sociopsicológicos de la comunicación pedagógica y su importancia para el trabajo docenteeducativo. Material Mimeografiado. La Habana: CEPES, U. H; 1999

Parra, Maria Eugenia (1998) "La etnografía de la educación”, en Cinta de Moebio, Núm. 3, Abril 1998. Facultad de Ciencias Sociales, Universidad de Chile. Artículo en línea, disponible en http://www.moebio.uchile.cl/03/frprin04.htm (Fecha de consulta: octubre 2006).

Pérez Escoda, N., Filella Guiu, G., \& Bisquerra Alzina, R. (2009). A los 100 años de la orientación: de la orientación profesional a la orientación psicopedagógica.

Riesgo, D. S., Jayme, M., \& Ordaz, M. I. C. (2018). La preparación a maestros primarios para la formación vocacional y orientación profesional hacia carreras agropecuarias. Mendive, 16(1), 20-38.

Santana Sardi, G. A., \& Vigueras Moreno, J. A. (2019). Hacia un Sistema Virtual de orientación vocacional. Revista Cubana de Educación Superior, 38(3).

Santana-Sardi, G. A., Vélez-Vélez, M. E., Sampedro-Mera, M. J., \& DelgadoGarcía, Y. S. (2020). Estrategia de formación vocacional orientada a la admisión estudiantil de educación superior, una perspectiva psicopedagógica. Dominio de las Ciencias, 6(3), 776-796

TRILLA, Jaume. (1993a). La educación fuera de la escuela. Barcelona. Ariel. 276p.

Valdés Carrillo, A. G., Valdés Carrillo, A. M., \& Fernández Oliva, B. (2016). Estrategia pedagógica para elevar la formación vocacional y orientación profesional de los estudiantes de Enfermería en la Facultad" Gral. Calixto García", 2013-2014. Educación Médica Superior, 30(2), 0-0. 